\title{
On the Exchange Matrix for WZNW Model
}

\author{
L. D. Faddeev \\ Steklov Mathematical Institute, Leningrad, USSR
}

Dedicated to Res Jost and Arthur Wightman

\begin{abstract}
The connection between the exchange algebra in the SU(2) WessZumino Novikov-Witten model and the quantum group $S U(2)$ is discussed. It is shown that on the quasiclassical level this connection has the simple interpretation in terms of the Lie-Poisson action of $S U(2)$ on the chiral components of the fields in the WZNW model.
\end{abstract}

Nowadays a new tendency proclaims itself in $2 d$ conformal field theory. In contrast to the pure bootstrap program of the original proposal by Belavin, Polyakov and Zamolodchikov [1] it is based on more traditional methods of quantum field theory. We see the return of the lagrangian formulation with the beautiful geometric interpretation of corresponding action (see i.e. [2]), use of the functional methods [3], etc. In view of the exact solvability of CFT, it is natural to invoke the approach of quantum integrable models [4]. In fact, it was extensively used in the beginning of 80-ties in connection with the Liouville model [5-7]. Moreover, the quantum group attributes already appeared in these papers. Now, when the quantum group methods are coming to fashion it is only reasonable to return to this approach and take off the mystery from the relations between conformal field theory and quantum groups.

A recent preprint by Gervais [8] on the Liouville model is one of the first steps in this direction. My note contains some results obtained independently. I have taken as a representative example the $S U(2)$ Wess-Zumino-Novikov-Witten model rather than Liouville (which is a reduction of noncompact $\operatorname{sl}(2, \mathbf{R}) \mathrm{WZNW}$ ) because the peculiarity of the elliptic monodromy is clearer there.

I shall work in the hyperbolic space time with the simplest topology of a cylinder,

$$
0 \leqq x \leqq 2 \pi ; \quad-\infty<t<\infty .
$$

The classical field variable $g(x, t)$ is a $2 \times 2$ unitary unimodular matrix, periodic in $x$

$$
g(x+2 \pi, t)=g(x, t) .
$$


The action functional

$$
A=\frac{1}{16 \gamma} \int \operatorname{tr}\left(\partial_{\mu} g g^{-1}\right)^{2} d x d t+\frac{1}{24 \gamma} \int \operatorname{tr} d^{-1}\left(d g g^{-1}\right)^{3}
$$

leads to the equation of motion

with solution

$$
\partial_{+}\left(g^{-1} \partial_{-} g\right)=0
$$

$$
g(x, t)=u(x+t) v(x-t)
$$

and Poisson bracket relations most readily written in terms of currents

namely

$$
R_{+}=u^{\prime} u^{-1} ; \quad L_{-}=-v^{-1} v^{\prime},
$$

$$
\begin{gathered}
\left\{R_{+}(x) \stackrel{\otimes}{,} R_{+}(y)\right\}=\gamma r \delta^{\prime}(x-y)+\frac{\gamma}{2}\left[R_{+}(x) \otimes I-I \otimes R_{+}(x), r\right] \delta(x-y), \\
\left\{L_{-}(x) \stackrel{\otimes}{\otimes} L_{-}(y)\right\}=-\gamma r \delta^{\prime}(x-y)+\frac{\gamma}{2}\left[L_{-}(x) \otimes I-I \otimes L_{-}(y), r\right] \delta(x-y) .
\end{gathered}
$$

Here $u$ and $v$ are unitary unimodular matrices $\left(R_{+}\right.$and $L_{-}$being antihermitian,

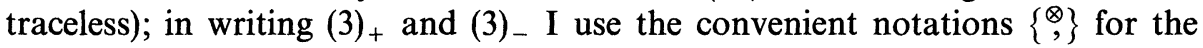
Poisson brackets of matrices with an evident definition; $4 \times 4$ matrix $r$ is given by

where $\sigma_{i}$ are Pauli matrices.

$$
r=\sum_{i} \sigma_{i} \otimes \sigma_{i}
$$

Matrices $u(x)$ and $v(x)$ will be called the chiral components. They can be also called chiral vertex operators, when quantized. If we normalize them by some appropriate initial conditions in the equations

$$
u^{\prime}=R_{+} u ; \quad v^{\prime}=-v L_{-}
$$

then the monodromy - an elliptic matrix $S$ such that

$$
u(x+2 n)=u(x) S ; \quad v(x+2 n)=S^{-1} v(x)
$$

is a dynamical variable.

All this is very well known and explicitly explained by Witten in [9]. However the natural question about the brackets of matrices $u$ and $v$ as well as their quantization was not answered until recently. Led by the Liouville example investigated by Gervais and Neveu [6] with important clarification by Babelon [10], one could suspect that these brackets must look like this (in the following we shall consider only one chiral component, say $u(x)$ )

$$
\left\{u(x)^{\otimes}, u(y)\right\}=u(x) \otimes u(y) q^{ \pm},
$$

where \pm correspond to $x>y$ and $x<y$ and $q^{ \pm}$being classical $r$-matrices,

$$
q^{+}=\frac{1}{2} \gamma\left(\begin{array}{rrrr}
-1 & 0 & 0 & 0 \\
0 & 1 & -4 & 0 \\
0 & 0 & 1 & 0 \\
0 & 0 & 0 & -1
\end{array}\right)
$$


and

$$
q^{-}=-P q^{+} P \text {. }
$$

Here $P$ is a permutation matrix, $P=\frac{1}{2}(I+r)$. Some arguments were given recently by Block [11] who used the canonical variables introduced in [2].

However these relations being good for the $\operatorname{sl}(2, \mathbf{R})$ case cannot be used directly in the unitary case. Indeed, if $u(x)$ is unitary in (5) then $q^{ \pm}$are to be hermitian $4 \times 4$ matrices.

The quantum generalization of (5)

$$
P u(x) \otimes u(y)=u(y) \otimes u(x) Q^{ \pm}
$$

where $Q$ is a $\operatorname{sl}(2) R$-matrix

and

$$
Q^{+}=\left(\begin{array}{cccc}
e^{i \gamma / 2} & 0 & 0 & 0 \\
0 & e^{i \gamma / 2}-e^{-3 i \gamma / 2} & e^{-i \gamma 2} & 0 \\
0 & e^{-i \gamma / 2} & 0 & 0 \\
0 & 0 & 0 & e^{i \gamma / 2}
\end{array}\right)
$$

$$
Q^{-}=\left(Q^{+}\right)^{-1}
$$

has the same drawback: in $s u(2)$ case the matrix $Q$ is to be unitary.

The way out of this difficulty is to observe, that relations (5) or (6) depend on the boundary conditions for solving Eq. (4). In particular, for the Floque solution $u^{F}$ with the diagonal monodromy

$$
S=\left(\begin{array}{cc}
e^{i p} & 0 \\
0 & e^{-i p}
\end{array}\right), \quad 0 \leqq p \leqq \pi,
$$

the matrix $Q$ must depend on the quasimomentum $p$. We can hope that among such admissible matrices we are able to find a suitable one. The message of this paper is that it is indeed the fact.

I shall discuss the quantum version from the beginning. Suppose that 1. The matrix elements of $u^{F}(x)$ have simple commutation relations with quasimomentum: for any arbitrary function $f(p)$ we have

$$
\begin{aligned}
& u_{i 1}^{F}(x) f(p)=f(p-\gamma) u_{i 1}^{F}(x), \\
& u_{i 2}^{F}(x) f(p)=f(p+\gamma) u_{i 2}^{F}(x),
\end{aligned}
$$

$i=1,2$. (The reason will be clarified later.)

2. Matrix $u^{F}(x)$ satisfies the commutation relations

$$
P u^{F}(x) \otimes u^{F}(y)=u^{F}(y) \otimes u^{F}(x) Q_{F}^{ \pm}(p)
$$

with unknown unitary matrix $Q_{F}^{ \pm}(p)$ and a linear combination

$$
u=u^{F} \Omega(p)
$$

satisfies the relation (6) with $Q^{ \pm}$given by (7).

It turns out that these conditions define $\Omega$ and $Q_{F}^{ \pm}(p)$ almost uniquely.

Indeed, substituting (11) into (6) and using (10) we find relation

$$
M(p) Q^{ \pm}=Q_{F}^{ \pm}(p) M(p),
$$


where $4 \times 4$ matrix $M(p)$ is written in terms of the matrix elements of matrix $\Omega$

as follows

$$
\Omega=\left(\begin{array}{ll}
\alpha & \beta \\
\gamma & \delta
\end{array}\right)
$$

$$
M=\left(\begin{array}{cccc}
\alpha_{+} \alpha & \alpha_{+} \gamma & \gamma_{+} \alpha & \gamma_{+} \gamma \\
\alpha_{-} \beta & \alpha_{-} \delta & \gamma_{-} \beta & \gamma_{-} \delta \\
\beta_{+} \alpha & \beta_{+} \gamma & \delta_{+} \alpha & \delta_{+} \gamma \\
\beta_{-} \beta & \beta_{-} \delta & \delta_{-} \beta & \delta_{-} \delta
\end{array}\right)
$$

Here $\alpha_{+}=\alpha(p+\gamma), \alpha_{-}=\alpha(p-\gamma)$, etc.

We shall look for $Q_{F}^{+}(p)$ in the form

$$
Q_{F}^{+}(p)=\left(\begin{array}{cccc}
f & 0 & 0 & 0 \\
0 & a & c & 0 \\
0 & b & d & 0 \\
0 & 0 & 0 & f
\end{array}\right)
$$

and chose $f=e^{i \gamma / 2}$ - the same as in $Q^{+}$. Then (12) gives 12 equations for 8 unknowns, $\alpha, \beta, \gamma, \delta, a, b, c, d$. Fortunately some equations are dependent and we end with the result:

$$
\begin{gathered}
a(p)=\frac{\sin \gamma}{\sin p} e^{i\left(p-\frac{1}{2} \gamma\right)} ; \quad d(p)=a(-p) \\
b=e^{-i \gamma / 2} \frac{\sin (p+\gamma)}{\sin p} h(p) ; \quad c=e^{-i \gamma / 2} \frac{\sin (p-\gamma)}{\sin p} \frac{1}{h(p)}
\end{gathered}
$$

where

$$
h(p)=\frac{\alpha(p) \beta(p+\gamma)}{\alpha(p-\gamma) \beta(p)}
$$

is not fixed by the equations. We can choose it to make $Q_{F}^{+}$unitary so that $b$ and $c$ become

$$
b(p)=e^{-i \gamma / 2} \frac{\sqrt{\sin (p+\gamma) \sin (p-\gamma)}}{\sin p}, \quad c(p)=b(-p) .
$$

Matrix $Q_{F}^{-}$is given by $Q_{F}^{-}=\left(Q_{F}^{+}\right)^{-1}$.

It is gratifying to observe that the matrix $Q_{F}^{+}$obtained coincides with $6 j$ symbol of $s l_{q}(2)$ introduced by Kirillov and Reshetikhin in [12] for the spin 1/2 case if we identify

$$
q=e^{2 i \gamma} ; \quad p=(2 j+1) \gamma .
$$

Since this object (and its generalization to other groups and representation) is the main ingredient of the modern treatment of the bootstrap program (see survey [13] and numerous literature there) its appearance in our treatment shows its most natural place in QFT: it is an exchange matrix for chiral component $u_{F}$ of a field operator $g(x, t)$ with diagonal monodromy $S$. 
The quantization $j=0,1 / 2, \ldots$ inherent in (14) is a natural consequence of the compactness of the portion of the phase space for $p$ and conjugate $q$, entering into $u^{F}$ as

$$
u_{i 1}^{F}=e^{i q} \tilde{u}_{i 1} ; \quad u_{i 2}^{F}=e^{-i q} \tilde{u}_{i 2}, \quad 0 \leqq q \leqq 2 \pi
$$

and having relation

with $p$.

$$
[p, q]=-i \gamma
$$

The component $v^{F}$ of opposite chirality with diagonal monodromy

$$
v^{F}(x+2 \pi)=\left(\begin{array}{cc}
e^{-i p} & 0 \\
0 & e^{i p}
\end{array}\right) v^{F}(x)
$$

satisfy the relation

$$
v^{F}(x) \otimes v^{F}(y) P=Q_{F}^{ \pm}(p) v^{F}(y) \otimes v^{F}(x) .
$$

Components $u^{F}$ and $v^{F}$ need not commute among themselves due to the appearance of $e^{ \pm i q}$ factors; however the relation of the form

$$
(v(x) \otimes I)(I \otimes u(y))=(I \otimes u(y)) C(v(x) \otimes I)
$$

ensures that the local field

$$
g(x)=u(x) v(x)
$$

(defined with an appropriate renormalization) is commutative.

I finish with several comments.

1. The main formula (10) makes it clear how the quantum group acts in QFT. Writing down (1) as

$$
g_{i k}(x, t)=\sum_{\alpha} u_{i \alpha}(x+t) v_{\alpha k}(x-t)
$$

with enforced distinction in writing the indices we can say that the quantum group acts on the $\alpha$-index. This action is seen only after the chiral components are introduced and it is hidden when one considers only local field variables (cf. [14, 15]).

2. We did not give any direct derivation of the relation (10). To do it one is to make control of the quantum expressions of the sort

$$
u=P \exp \int_{0}^{x} R_{+}(x) d x u(0)
$$

with proper quantization of the classical relation (3) $)_{+}$. Instead several consistency checks are available.

i. From (10) it follows, that if $f_{+}=e^{i \gamma / 2}$ and

then

$$
u_{11}^{F}(2 \pi)=u_{11}^{F}(0) e^{i p},
$$

$$
e^{i p} u_{11}^{F}(x)=u_{11}^{F}(x) e^{i(p+\gamma)}
$$

which (with analogous formulas for other matrix elements) confirms (8). 
ii. Classical limit of (10) looks as follows

$$
\left\{u^{F}(x)^{\otimes}, u^{F}(y)\right\}=u^{F}(x) \otimes u^{F}(y) q_{F}^{ \pm}(p),
$$

where $q_{F}^{ \pm}(p)$ is given by quasiclassical expansion of $Q_{F}^{ \pm}(p)$

$$
Q_{F}^{ \pm}(p)=P\left(1-i q_{F}^{ \pm}(p)+0\left(\gamma^{2}\right)\right)
$$

and have the form

$$
q_{F}(p)=\frac{\gamma}{2}\left(\begin{array}{cccc}
-\varepsilon & 0 & 0 & 0 \\
0 & \varepsilon & -2(\varepsilon+i \operatorname{ctg} p) & 0 \\
0 & -2(\varepsilon-i \operatorname{ctg} p) & \varepsilon & 0 \\
0 & 0 & 0 & -\varepsilon
\end{array}\right)
$$

where $\varepsilon=\varepsilon(x-y)$. The differential equation, satisfied by $q_{F}$

$$
q_{x y}^{F}=-\gamma r \delta^{\prime}(x-y)
$$

ensures that $R_{+}$introduced in (2) satisfies the relations (3).

iii. The Yang-Baxter relation, satisfied by $Q^{ \pm}$makes the Poisson bracket or commutation relations consistent (Jacobi identity or associativity).

iv. It is plausible that the properties listed above define $Q_{F}(p)$ essentially uniquely.

3. In the Liouville model the role of Eq. (4) is played by

$$
\psi^{\prime \prime}+S \psi=0
$$

where $S(x)$ comprises the generators of the Virasoro algebra, i.e. classically

$$
\{S(x), S(y)\}=\gamma(S(x)+S(y)) \delta^{\prime}(x-y)+\gamma \delta^{\prime \prime \prime}(x-y) .
$$

For two linearly independent solutions $\psi_{1}, \psi_{2}$ combined in a row $\psi=\left(\psi_{1}, \psi_{2}\right)$ we should have (in quantum version)

$$
\psi(x) \otimes \psi(y)=\psi(y) \otimes \psi(x) Q^{ \pm},
$$

where $Q^{ \pm}$coincide with (7) for a particular choice of $\psi$. For other choices $Q$ will depend on $p$.

In the original paper of Gervais and Neveu the matrix $Q(p)$ appeared corresponding to the choice

$$
h(p)=-\frac{\Gamma(p+\gamma / \pi) \Gamma(1-p / \pi)}{\Gamma(-p+\gamma / \pi) \Gamma(1+p / \pi)} .
$$

Recently Gervais preferred the choice $h=1$. However for the effective $s u(2)$ case $(c<1$ or $0 \leqq \gamma \leqq \pi)$ when the quasimomentum entering through

$$
\psi_{1}(x+2 \pi)=\psi_{1}(x) e^{i p}
$$

is real the choice corresponding to my $Q_{F}(p)$ is more appropriate. In the $\operatorname{sl}(2, \mathbf{R})$ hyperbolic case $(c>25$ or $-\pi \leqq \gamma \leqq 0) p$ must be changed $p \rightarrow i p$ (I don't agree here with Gervais [8]) and the classical analoque of (16) with $Q=Q_{F}$ follows from identification $[6,7,15]$,

$$
\psi_{1}=\left(u^{\prime}\right)^{-1 / 2} ; \quad \psi_{2}=u\left(u^{\prime}\right)^{-1 / 2},
$$


where $u$ have Poisson brackets

$$
\{u(x), u(y)\}=\gamma \varepsilon(x-y)) u(x)-u(y))^{2}+\gamma \operatorname{cth} p\left(u^{2}(x)-u^{2}(y)\right) .
$$

Note that the quantum lattice analogues are also proposed in $[7,15]$. It is still unclear which $\gamma$ correspond to the $s(2, \mathbf{R})$ elliptic case. Naive continuation hints that $\gamma$ is complex, $|\gamma|=\pi$.

4. Due to the simple relation

$$
u_{11}(x) u_{11}(y)=u_{11}(y) u_{11}(x) e^{i \gamma \varepsilon(x-y)}
$$

it is usually proposed to parametrize $u_{11}$ in terms of a free field (oscillators and zero modes). This is essentially the origin for free fields in CTF. However it seems to me that the quantum group relations (10) are to be used directly for the calculation of the Green functions. The implementing of such a program is now in progress.

5. The expression under the square root in (13) vanishes for $p=\gamma$ and $p=\pi-\gamma$. This shrinks the interval for admissible $\pi$ from $p$ to $\pi-2 \gamma$. It can be compared with the famous renormalization $\gamma \rightarrow \pi \gamma / \pi-\gamma$ characteristic of the Sine-Gordon model (see $[17,18])$. This also explains why $p$ gets $K+1$ different values when $\gamma$ is given by $\gamma=\frac{\pi}{K+2}$, namely $j=0, \frac{1}{2}, \ldots \frac{K}{2}$ in (14).

6. It is worthwhile to mention that CFT models should be understood as the particular limits of quantum magnetic chain models, delat with in the general scheme of algebraic Bethe-Ansatz [4]. The works of Volkov [16, 19] are indications of this in the Liouville case. The works of F. Smirnoff [20] show the same from another point of view.

Acknowledgements. This work was begun and essentially finished during may stay in Italy in October 1989. I appeciate the hospitality and excellent working conditions provided by Professors L. Radicati, R. Pittorino and A. Salam in Scuola Normale di Pisa, Institute of Physics of the University of Naples and ICPT in Trieste, correspondingly. I am grateful to A. Alekseev, O. Babelon, A. Kirillov, F. Smirnoff, and S. Shatashvili for discussions.

\section{References}

1. Belavin, A., Polyakov, A., Zamolodchikov, A.: Nucl. Phys. B 241, 333 (1984)

2. Alekseev, A., Shatashvili, S.: Nucl. Phys. B 323, 719 (1989)

3. Alekseev, A., Shatashvili, S.: LOMI preprint E-7-89

4. Faddeev, L.: In: Recent Advances in field theory. Stora, R., Zuber, J.B. (eds.). Amsterdam: North-Holland 1984

5. Gervais, J.-L., Neveu, A.: Nucl. Phys. B 224, 329 (1983)

6. Gervais, J.-L., Neveu, A.: Nucl. Phys. B 238, 125 (1984)

7. Faddeev, J., Takhtajan, L.: Lecture Notes in Phys., vol. 246, p. 166. Berlin, Heidelberg, New York: Springer 1986

8. Gervais, J.-L.: preprint Ecole Normale LPTENS 89/14, 1989

9. Witten, E.: Commun. Math. Phys. 92, 455 (1984)

10. Babelon, O.: Phys. Lett. B 215, 523 (1988)

11. Block: preprint Tel-Aviv University, 1989

12. Kirillov, A., Reshetikhin, N.: LOMI preprint E-9-88

13. Moore, G., Seiberg, N.: preprint Rutgers University RU-89-32 
14. Moore, G., Reshetikhin, N.: Preprint Institute for Advanced Study IASSNS-HEP 89/18

15. Reshetikhin, N., Smirnoff, F.: Preprint

16. Volkov, A.: Zap. Nauch. Sem. LOMI 151, 24 (1987) (to be translated in Sov. J. Math.)

17. Dashen, R., Hasslaher, B., Neveu, A.: Phys. Rev. D 11, 3424 (1975)

18. Sklyanin, E., Takhtajan, L., Faddeev, L.: Theor. Math. Phys. 40, 194 (1979)

19. Volkov, A.: Zap. Nauch. Sem. LOMI 150, 17 (1986) (to be translated in Sov. J. Math.)

20. Smirnoff, F.: LOMI preprint E-4-89

Communicated by N. Yu. Reshetikhin

Received December 20, 1989 